\title{
Parametrização de Sinais de EEG para Diagnóstico de Epilepsia Usando Matrizes de Kernel
}

\author{
$1^{\text {st }}$ Paulo Cirillo S. Barboza \\ Departamento de Engenharia de Teleinformática (DETI) \\ Universidade Federal do Ceará (UFC) \\ Centro de Tecnologia, Fortaleza \\ paulocir@alu.ufc.br
}

\author{
$2^{\text {st }}$ Guilherme de Alencar Barreto \\ Departamento de Engenharia de Teleinformática (DETI) \\ Universidade Federal do Ceará (UFC) \\ Centro de Tecnologia, Fortaleza \\ gbarreto@ufc.br
}

\begin{abstract}
Resumo-O presente trabalho aborda o processo de extração de atributos de sinais de eletroencefalograma (EEG), também chamado de parametrização de sinais, para o auxílio na deteç̧ão de crises epiléticas. A abordagem proposta neste trabalho é baseada na utilização de matrizes kernel para parametrizar segmentos do sinal de EEG de modo a construir vetores de atributos rotulados. Para isso, técnicas de esparsificação são usadas a fim de diminuir a dimensão das matrizes de kernel. Além disso, novas estratégias para a construção das matrizes de kernel e, consequentemente dos vetores de atributos resultantes, são também exploradas com base em três hipóteses para segmentação dos sinais de EEG e na análise isolada (intra-canal) ou conjunta (inter-canais) dos canais de EEG. Um amplo estudo comparativo avaliando o desempenho dos métodos propostos a partir da variação dos seus hiperparâmetros é executado. A partir da análise e discussão dos resultados obtidos, foi possível verificar que os métodos propostos são promissores, apresentando desempenho equivalente ou superior aos gerados por métodos clássicos de extração de atributos de sinais de EEG.

Index Terms-EEG, Epilepsia, Aprendizagem de máquina, Extração de atributos, Matrizes de kernel.
\end{abstract}

\section{INTRODUÇÃo}

Tratando-se de aplicações de Aprendizado de Máquina (AM) para o setor da saúde, especificamente dentro da medicina, pode-se elencar pesquisas científicas que utilizam sinais biopotenciais para resolver problemas associados ao reconhecimento de padrões [1]. Dentre esses sinais, destaca-se o de Eletroencefalograma (EEG), que monitora as atividades cerebrais através de eletrodos posicionados no couro cabeludo [2]. Estes sinais são amplamente utilizados, uma vez que possuem uma alta resolução temporal e método de aquisição não invasivo. Além disto, desempenham um papel vital em diversas aplicações de modo a melhorar a qualidade de vida de pessoas com doenças cerebrais [3]. O EEG é utilizado como ferramenta facilitadora para diagnósticos de doenças como Alzheimer, tumores cerebrais, distúrbios de sono, epilepsia, dentre outros [4].

A epilepsia, de acordo com a International League Against Epilepsy (ILAE) e a International Bureau for Epilepsy (IBE), é uma doença cerebral presente no paciente que já teve uma convulsão e, por alguma razão, seu cérebro possui uma tendência recorrente a ter novos episódios convulsivos [5]. Tais convulsões são caracterizadas como atividades cerebrais excessivas e anômalas, que podem causar perdas de consciência, memória ou respiração [4]. A análise de sinais de EEG é uma ferramenta de grande importância no auxílio da detecção de crises epiléticas, porém, este processo além de necessitar de muito tempo de monitoramento do paciente, também requer do médico uma acurada habilidade de observação ao realizar a inspeção visual dos sinais necessários para identificar corretamente os episódios cerebrais epiléticos. Esses períodos são classificados em quatro categorias: ictal (período de crise convulsiva); pós-ictal (período após a crise, em que podem ser observados sintomas como dores de cabeça, confusão e náusea); pré-ictal (período que precede uma futura crise); interictal (qualquer período entre crises) [7].

Estudos para detecção de crises convulsivas constituem uma área bastante ativa de AM e processamento de sinais. Usualmente, tal tarefa requer várias etapas, tais como aquisição do sinal, pré-processamento, extração e seleção de atributos e, por fim, classificação [8]. Porém, esta tarefa não é simples, em virtude da natureza não linear, não estacionária e estocástica dos sinais de EEG [9], [10]. Além disso, como esses sinais operam em frequências muito baixas $(<30 \mathrm{~Hz})$, há uma grande suscetibilidade a ruídos e artefatos, que podem ser provenientes de outros biossinais, de interferência causada pela rede elétrica e de falhas ou mau posicionamento dos eletrodos [11]. Assim, em face de tais dificuldades, a etapa de extração de atributos apresenta-se como fundamental para o sucesso da tarefa de detecção de epilepsia, uma vez que é a responsável por extrair as informações úteis do sinal de EEG [6].

O presente trabalho tem por objetivo avaliar a utilização de matrizes kernel para parametrizar segmentos do sinal de EEG com o intuito de montar vetores de atributos rotulados, vetores estes que serão usados no treinamento de classificadores para detectar a presença ou ausência de crises epiléticas. Para isso, técnicas de esparsificação são usadas para diminuir a dimensão das matrizes de kernel. Além disso, novas estratégias para a construção das matrizes de kernel e, consequentemente dos vetores de atributos resultantes, são também exploradas com base em três hipóteses para segmentação dos sinais de EEG e na análise isolada (intra-canal) ou conjunta (inter-canais) dos canais de EEG. 


\section{A. Trabalhos Relacionados}

No estudo realizado em [12], em que foram realizadas análises do desempenho da extração de atributos nos domínios do tempo, frequência e em ambos. Realizaram-se análises comparativas do desempenho do classificador Naive Bayes em relação aos métodos propostos e os autores do estudo observaram que ao se utilizar variância, energia, energia não linear e entropia de Shannon extraídos do sinal de EEG, observou-se uma boa distinção de sinais convulsivos dos normais [12]. O estudo reportado em [4] abordou a detecção de crises epiléticas realizando a extração de atributos em duas etapas. Inicialmente aplicou-se a STFT e, em seguida, três diferentes métodos. $\mathrm{O}$ primeiro deles baseou-se em frequência; o segundo na aplicação de $K$-médias e, por último, realizou-se a análise de picos máximos. Na etapa de classificação foram utilizados os classificadores SVM, MLP e $k$-NN, com destaque para a acurácia de 99,5\% da rede MLP [4]. No estudo reportado em [13] foram utilizados quatro métodos de extração de atributos (coeficientes LPC, coeficientes mel-cepstrais (MFCC, na sigla em inglês), covariância e densidade espectral de potência e seus desempenhos para cinco algoritmos de classificação. Os coeficientes LPC e MFC apresentaram os melhores desempenhos, enquanto o método de covariância apresentou com a melhor relação entre desempenho e custo computacional.

\section{Métodos Clássicos de Extração de Atributos}

Nesta seção são apresentados dois métodos clássicos utilizados no trabalho para realizar a extração dos atributos dos sinais de EEG. A proposta sugerida, segue de acordo com o fluxograma resumido ilustrado pela Figura 1. Nesta, é possível identificar que alguns métodos são aplicados tanto na análise inter-canais como para a intra-canal, com a adequação para tal feito, descrita na seção III. Além disto, pode-se visualizar que a aplicação do método LPC é realizada apenas pela abordagem intra-canais.

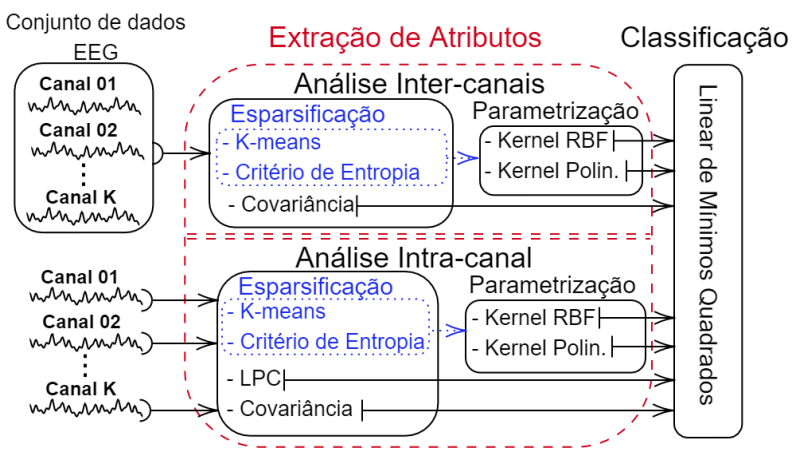

Figura 1. Fluxograma da proposta sugerida

\section{A. Método da Covariância}

Para verificar como os sinais de EEG obtidos dos vários eletrodos (i.e., canais) são estatisticamente relacionados, podese utilizar a matriz de covariância como extrator de atributos.
Ao se trabalhar com sinais de EEG, normalmente o equipamento que faz a aquisição dispõe de uma quantidade $K$ de canais, e como as crises epiléticas podem ser generalizadas, é conveniente o uso da matriz de covariância, pois, ela permite uma análise espacial inter-canais. Esta matriz, simétrica e de dimensões $K \times K$, carrega informações de variância entre atributos em sua diagonal principal e covariância de pares, nos demais elementos [14], [19].

Para aplicar a técnica da matriz de covariância faz-se necessário definir um intervalo de duração do segmento de EEG (chamado de época no jargão da área), que com a taxa de amostragem vai definir o número de amostras do sinal dentro da época. Por exemplo, para uma época de $2 \mathrm{~s}$ de duração, a uma taxa de amostragem de $256 \mathrm{~Hz}$, tem-se um segmento de 512 amostras para um canal. Considerando $K$ canais, tem-se a cada instante de amostragem $t$ dentro da época, um vetor de atributos $\mathbf{x}(t) \in \mathbb{R}^{K \times 1}$ formado pelas leituras das amplitudes dos sinais de EEG de cada um dos $K$ canais.

Isto posto, a matriz de covariância $\hat{\mathbf{C}}_{\mathbf{x}}$ pode ser estimada pela seguinte expressão:

$$
\hat{\mathbf{C}}_{\mathbf{x}}=\frac{1}{N} \sum_{t=1}^{N}[\mathbf{x}(t)-\mathbf{m}][\mathbf{x}(t)-\mathbf{m}]^{T}
$$

na qual $\mathbf{m} \in \mathbb{R}^{K \times 1}$ é o vetor centróide dos $N$ vetores de atributos [19].

Por fim, a cada época transcorrida, deve-se associar um vetor de atributos que herdará o rótulo do estado do paciente (i.e., em crise ou não) para aquele período em análise. Como o classificador de padrões avaliado é treinado/testado com vetores de atributos em vez de matrizes, a matriz de covariância associada a uma dada época deverá ser vetorizada. Como a matriz $\hat{\mathbf{C}}_{\mathbf{x}}$ é simétrica, constrói-se o vetor de atributos usando-se apenas os elementos da diagonal principal (variâncias) e os elementos da matriz triangular superior (covariâncias). As covariâncias devem ser multiplicadas por $\sqrt{2}$ para preservar a norma de Frobenius da matriz de covariância original [19]. O vetor de atributos resultante terá então dimensão $(K+1) K / 2 \times 1$.

\section{B. Coeficientes LPC}

O filtro autorregressivo (AR) é amplamente utilizado em análises de séries temporais, e é uma função que modela $p$ valores passados de uma série [15]. Sinais de EEG podem ser parametrizados pelos coeficientes do filtro AR, estimados dentro de uma época, já que para trechos curtos do sinal de EEG pode-se assumir estacionariedade. Esta técnica é conhecida, portanto, como coeficientes da Codificação Linear Preditiva (LPC, do inglês Linear Predictive Coding) e, diferentemente do método da covariância, é aplicada a um canal por vez.

Considerando um sinal de tempo discreto $\{x(1), x(2), \cdots, x(N)\}$, o filtro AR de ordem $p$ é definido como [16]

$$
x(t)=a_{1} x(t-1)+a_{2} x(t-2)+\cdots+a_{p} x(t-p)+v(t)
$$

em que $p$ é a ordem do filtro, $a_{1}, a_{2}, \cdots, a_{p}$ são os coeficientes LPC e $v(n)$ representa um processo de ruído branco gaussiano. 
Para utilizar este método como extrator de atributos, deve-se especificar a ordem $p$ e estimar os coeficientes correspondentes. No presente artigo, utilizou-se o método de estimação Linear de Mínimos Quadrados (OLS, do inglês Ordinary Least Squares). Para isso, usando as amostras do segmento de tamanho $N$ do sinal de EEG, montam-se o vetor de predições $\mathbf{p} \in \mathbb{R}^{(N-p) \times 1}$ e a matriz de regressores $\mathbf{X} \in \mathbb{R}^{(N-p) \times p}$ como segue

$$
\mathbf{p}=\left[\begin{array}{c}
x(p+1) \\
x(p+2) \\
\vdots \\
x(N)
\end{array}\right] \text { e } \mathbf{X}=\left[\begin{array}{cccc}
x(p) & x(p-1) & \cdots & x(1) \\
x(p+1) & x(p) & \cdots & x(2) \\
\vdots & \vdots & \ddots & \vdots \\
x(N-1) & x(N-2) & \cdots & x(N-p)
\end{array}\right]
$$

Assim, o vetor de coeficientes LPC para um dado canal e para a época atual é estimado por meio da seguinte expressão:

$$
\hat{\mathbf{a}}=\left(\mathbf{X}^{T} \mathbf{X}\right)^{-1} \mathbf{X}^{T} \mathbf{p} .
$$

Este procedimento se repete para cada canal de forma isolada e independente. Ao final, o vetor de atributos da época atual é formado pela concatenação dos vetores de coeficientes LPC dos $K$ canais, o que resulta em um vetor de dimensão $(K \cdot p) \times 1$.

\section{ABordagens PROpostas}

Serão descritos a seguir os métodos propostos para a extração de atributos do sinal de EEG baseados em matrizes de kernel e as técnicas de esparsificação para redução da dimensão das matrizes de kernel. Inicialmente, será feita a definição clássica da matriz de kernel, fundamental para o entendimento deste artigo. Será apresentada a descrição do conjunto de dados utilizado para validar as propostas apresentadas neste artigo e como o mesmo foi particionado. Em sequência, será especificado como cada método é utilizado nos dois caminhos investigativos, ou seja, via análise intra-canal ou inter-canais.

\section{A. Matriz de Kernel}

O conceito de matriz de kernel é amplamente utilizado na área de reconhecimento de padrões, porém normalmente utiliza-se no projeto de classificadores baseados em máquinas de vetores suporte. Este conceito tenta resolver problemas não lineares convertendo-os em lineares, através de uma transformação para um espaço de características com alta dimensão ou até infinita. Portanto, ao se aplicar essa transformação em um padrão de entrada $\mathbf{x} \in \mathbb{R}^{n}$ resultará em $\phi(\mathbf{x}) \in \mathbb{R}^{m}$, na qual $m$ é a dimensão do espaço de Hilbert de reconstrução.

Uma matriz de kernel $\mathbf{K}=\left[K_{i j}\right]_{N \times N}$, em que $N$ é o número de exemplos de treinamento, é uma matriz gramiana, ou seja, uma matriz cujo elemento $K_{i j}$ é o produto interno dos vetores $\mathbf{x}_{i}$ e $\mathbf{x}_{j}$ quando mapeados no espaço de Hilbert pela função $\phi(\cdot, \cdot)$, de maneira que: $K\left(\mathbf{x}_{i}, \mathbf{x}_{j}\right)=\left\langle\phi\left(\mathbf{x}_{i}\right), \phi\left(\mathbf{x}_{j}\right)\right\rangle=$ $\phi\left(\mathbf{x}_{i}\right)^{T} \phi\left(\mathbf{x}_{j}\right)$ [18]. No entanto, a função $\phi(\cdot, \cdot)$ é usualmente desconhecida, e portanto, utilizam-se funções genéricas que reproduzem o produto interno no espaço de Hilbert de reconstrução. Esta estratégia é conhecida como truque do kernel, sendo que a função polinomial $\left(\mathbf{x}_{i}, \mathbf{x}_{j}\right)=\left(\mathbf{x}_{i}^{T} \mathbf{x}_{j}+c\right)^{d}$, em que $d$ é a ordem do polinômio e $c$ é um parâmetro livre, e a função gaussiana (ou RBF) $\left(\mathbf{x}_{i}, \mathbf{x}_{j}\right)=\exp \left(-\frac{\left\|\mathbf{x}_{i}-\mathbf{x}_{j}\right\|^{2}}{2 \sigma^{2}}\right)$, em que $\sigma$ é a largura da gaussiana, são escolhas comuns em aplicações práticas [18]. A matriz gramiana resultante do uso dessas funções kernel deve ser positiva definida.

\section{B. Descrição do conjunto de dados utilizado}

Os experimentos conduzidos neste trabalho foram aplicados ao conjunto de dados público CHB-MIT Scalp EEG, que é composto por sinais de EEG coletados de pacientes pediátricos com convulsões intratáveis, sendo cada paciente um possível candidato à intervenção cirúrgica [17]. Em tal base de dados, o monitoramento foi realizado durante diversos dias e sem alguma medicação preventiva contra convulsões. Neste conjunto de dados, as gravações foram divididas em 23 casos coletados de 22 pacientes. A explicação disto é pelo fato de que os casos chb01 e chb21 foram obtidos do mesmo paciente, com diferença entre as aquisições de um ano e meio. Dos 22 pacientes, cinco eram do sexo masculino e 17 do sexo feminino e suas idades variavam entre 1,5 até 19 anos. O autor relata ainda que após um ano do último experimento, um vigésimo quarto caso foi adicionado, porém este não possui informações sobre sexo e idade.

A aquisição do sinal de EEG foi realizada com uma taxa de amostragem de $256 \mathrm{~Hz}$, resolução de 16 bits e posicionamento de eletrodos de modo bipolar através do padrão 1020. Cada caso possuía entre nove a 42 arquivos com o sinal contínuo gravado, porém, destes arquivos apenas alguns possuem períodos ictais. Além disso, a maioria das aquisições foi realizada utilizando 23 canais, porém, em algumas situações utilizaram-se até 26 canais ou até mesmo outros tipos de sinais como Eletrocardiograma (ECG), ou estímulo do nervo vagal (VNS, do inglês Vagal Nerve Stimulus).

Para o presente trabalho buscou-se a correção das despadronizações mencionadas. Desta maneira, definiu-se que ao utilizar o conjunto de dados, apenas os 23 canais mencionados seriam aproveitados. Além disto, escolheram-se apenas os arquivos que possuíssem períodos ictais identificados. Em seguida, pensou-se numa maneira de explorar alguns conjuntos de treinamento e teste, a fim de mitigar efeitos transitórios dos períodos pré ou pós-ictal. Desta maneira, definiram-se três hipóteses de como subdividir o sinal completo. Estas hipóteses são ilustradas pela Figura 2, que apresenta um esboço das segmentações para cada hipótese. Os sinais em vermelho referem-se aos períodos ictais e os em azul aos sinais não ictais. Pode-se explicar ainda através da Figura 2, que nas hipóteses 1 e 2 a quantidade de dados ictais e não ictais são iguais e o segmento completo é contínuo. Isto se difere da hipótese 3 , na qual foi definido que o tamanho do sinal não ictal têm três vezes o tamanho do sinal ictal e possui um intervalo de 100 s no início e fim do período ictal.

\section{Esparsificação da matriz de dados}

A esparsificação é fundamentalmente a escolha e seleção de certa quantidade de vetores aleatórios de uma matriz de dados. 


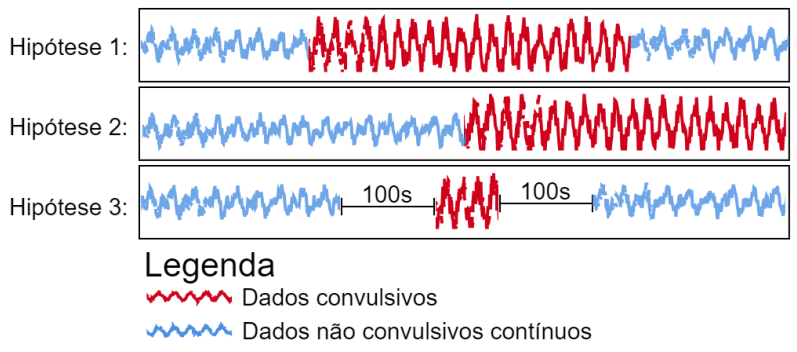

Figura 2. Hipóteses para geração de dados de treino e teste.

Esta nova matriz reduzida, é comumente chamada de dicionário de vetores relevantes. De modo a realizar uma seleção não aleatória dos vetores, escolheu-se a utilização de dois métodos. O primeiro, baseado em métodos de clusterização. Dentre os algoritmos disponíveis escolheu-se o $k$-médias, que tem o objetivo de encontrar $k$ grupos, de modo a minimizar a soma das distâncias entre ponto e centroides.

O segundo método utilizado em conjunto da extração de atributos é baseado na imposição de esparsificação através da escolha de $m$ vetores do conjunto de treinamento que possui tamanho $N$. A metodologia de aplicação pode ser visualizada através do Algoritmo 1, que necessita inicialmente da quantidade $m$ e que representa o tamanho do subconjunto. Em seguida, são computados dois subconjuntos $B_{i e}$ e $B_{a u x}$, sendo que o primeiro é composto por $m$ vetores do conjunto de dados original de modo aleatório e o segundo possui os elementos restantes não selecionados e portanto, sua ordem é $N-m$. Na sequência, de modo iterativo faz-se a troca de um padrão do conjunto $B_{i e}$ por um padrão em $B_{\text {aux }}$. Em cada iteração faz-se a estimação da matriz de kernel, calcula-se a entropia associada e caso seu valor seja superior à entropia anterior, este vetor é trocado. O procedimento repete-se até que o número máximo de iterações seja atingido [18].

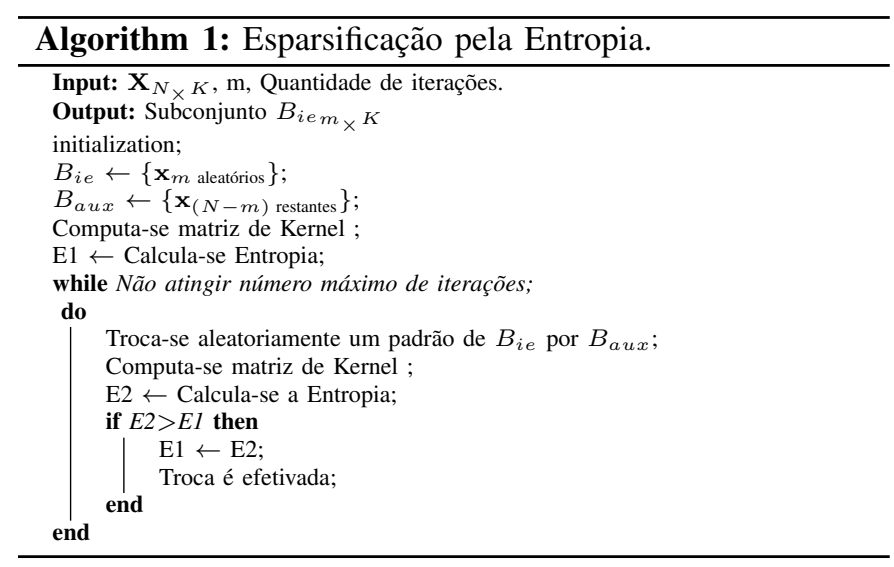

Para o critério de maximização de entropia, utilizou-se a entropia quadrática de Renyi aproximada, que é exibida pela Equação 5. Nesta Equação, $\boldsymbol{\Sigma}$ representa a matriz de kernel à ser avaliada e $\mathbf{1}_{m}$ é um vetor unitário de ordem $m$ [18].

$$
E=-\log \left(\frac{1}{m^{2}} \cdot \mathbf{1}_{m}^{T} \cdot \boldsymbol{\Sigma} \cdot \mathbf{1}_{m}\right)
$$

\section{Modo de Extração Inter-canais}

Como dito anteriormente, é comum a análise de sinais de EEG em curtos intervalos de tempo. Esta abordagem é válida tanto para a etapa de treino como para a de teste. Esses intervalos de tempo são comumente chamados de épocas, e para fins deste trabalho utilizou-se o intervalo de 2s. Portanto, 512 dados são analisados em uma época.

Assim, para os métodos em que se assume o modo de extração inter-canais, em cada época realiza-se a análise em um dicionário de vetores com ordem $N=512 \times K=23$. De posse desta matriz, é realizada a composição do dicionário de vetores relevantes pelos métodos de esparsificação para que assim possa ser realizada a estimação da matriz de kernel como extrator de atributos. Deste modo, o processo para se obter o vetor c parametrizado referente a uma época, é ilustrado pelo fluxograma na Figura 3. Este vetor então é rotulado com a respectiva classe do segmento do sinal e o processo é repetido para as demais épocas, concatenando cada vetor $\mathbf{c}$ pelas colunas. Na Figura 3 é possível verificar que ao se aplicar o método de esparsificação, obtém-se um de ordem $M \times K$. Para fins deste trabalho, esta ordem está relacionada com o parâmetro do método de esparsificação. Ou seja, $M=k$ se utilizado o método $k$-Médias e $M=m$ se for utilizado o critério pela entropia de Renyi.

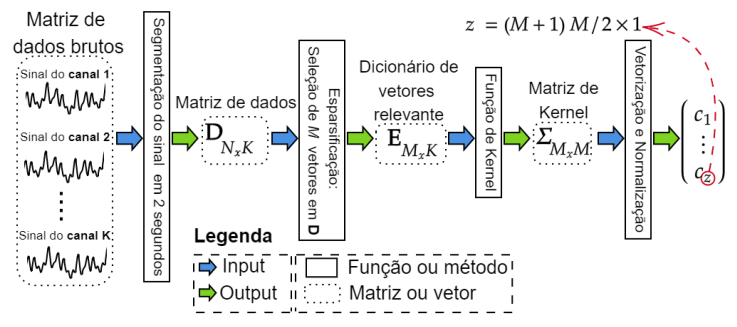

Figura 3. Fluxograma extração de atributos para uma época pela análise inter-canais

\section{E. Modo de Extração Intra-canal}

A análise dos sinais através desta proposta é feita de maneira diferente da anterior, porém, ainda se mantém a abordagem da esparsificação da matriz de dados. Para este caso, em que se assume o modo de extração intra-canal, o sinal é segmentado em uma época de 2 segundos e a parametrização é realizada de canal a canal, repetindo esse procedimento até o final da série temporal. Para formar a matriz de dados em uma época considerando um canal, ou seja $\mathrm{x} \in \mathbb{R}^{1 \times N=512}$, realiza-se a composição da matriz contendo os vetores de regressão de acordo com a Eq. (6). Nota-se que sua estimação é similar a Eq. (3), com a diferença de uma linha adicional, e portanto também necessita do parâmetro $p$ a ser definido.

$$
\mathbf{X}=\left[\begin{array}{cccc}
x(p) & x(p-1) & \cdots & x(1) \\
x(p+1) & x(p) & \cdots & x(2) \\
\vdots & \vdots & \ddots & \vdots \\
x(512) & x(512-1) & \cdots & x(512-p+1)
\end{array}\right]
$$


O processo para a parametrização pelo modo intra-canal de uma época é ilustrado pela Figura 4, na qual é possível verificar que em cada canal é realizado a parametrização separadamente, produzindo um vetor de ordem $z$. Esses vetores são concatenados a fim de produzir o vetor $\mathbf{v}_{(K=512 \cdot z)}{ }_{\times} 1$, que então é rotulado. O procedimento ilustrado é repetido até que o fim das épocas seja atingido.

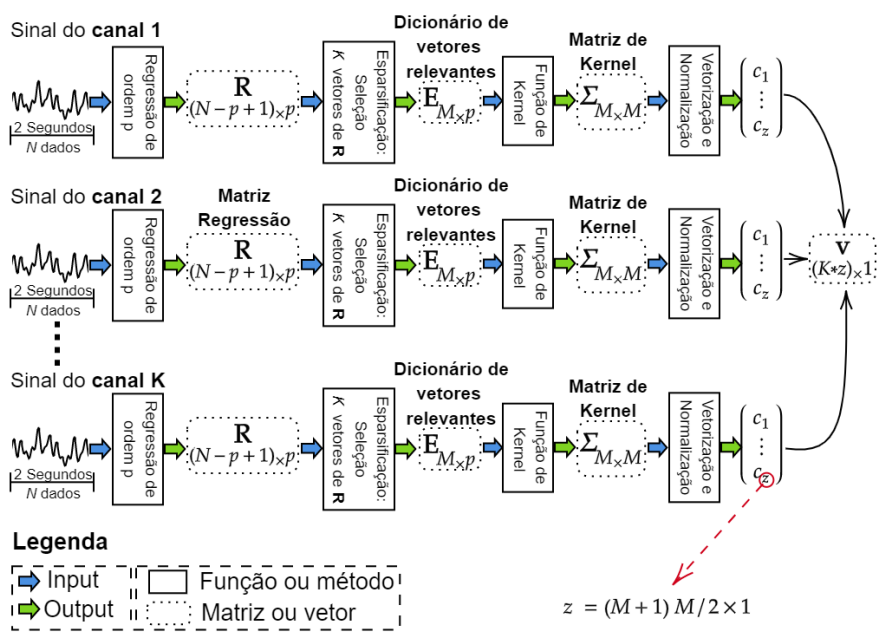

Figura 4. Fluxograma extração de atributos pela análise intra-canal

\section{F. Metodologia para etapa de classificação}

A fim de testar o desempenho de cada modelo gerado com os métodos aplicados, utilizou-se a validação cruzada nos arquivos do paciente em análise. A validação proposta é feita pela relação de um arquivo para teste e os demais para treino. Isto é repetido até que todos os arquivos sejam utilizados como teste.

Para compor a matriz de dados de treinamento, utilizou-se das informações mencionadas nas três hipóteses, bem como dos valores de início e fim dos períodos ictais, disponibilizados no conjunto de dados. Portanto, nesta etapa não se aplicam os métodos de parametrização em todo o sinal, mas sim apenas nos intervalos identificados como convulsivos e nos dados não convulsivos, utilizando os critérios de cada hipótese. Em seguida, faz-se a estimação dos modelos pelo algoritmo do OLS para cada uma das parametrizações realizadas. A etapa de teste se difere, pois, é realizada uma análise do arquivo por completo, ou seja, percorre-se o sinal do início ao fim, com avanços de 2 s. Em cada época faz-se a extração de atributos e em seguida o modelo faz a predição deste intervalo. Ao final das épocas são feitas as análises de desempenho para cada método de parametrização.

No presente trabalho, foram utilizadas as métricas de acurácia $\left(\frac{V P+V N}{V P+V N+F P+F N}\right)$, sensibilidade $\left(\frac{V P}{V P+F N}\right)$ e especificidade $\left(\frac{V N}{V N+F P}\right)$, para avaliar o desempenho dos métodos propostos. O problema de classificação é binário, portanto os dados são rotulados como +1 caso seja identificado um período ictal e -1 caso contrário. Pode-se representar através de uma matriz de confusão, de modo que, VP representa os verdadeiros positivos, $\mathrm{VN}$ os verdadeiros negativos, $\mathrm{FN}$ os falsos negativos e FP os falsos positivos. A partir das informações obtidas com a matriz de confusão é possível computar as métricas mencionadas.

Há uma problemática ao se analisar os resultados obtidos através destas equações. Isto se dá pelo fato de que a duração dos períodos ictais em qualquer arquivo é bastante inferior aos não ictais. Deste modo, procurou-se a adição de uma análise generalizada, calculando-se a média geométria das três métricas.

\section{RESUltados E Discuss Ão.}

Para compor os resultados do presente artigo, foi necessário inicialmente realizar uma exploração dos hiperparâmetros de cada método proposto. Definiu-se então que essa análise exploratória seria composta por arquivos do caso chb01, aplicandose apenas a segmentação pela hipótese 3 . O paciente em questão dispõe de sete arquivos contendo sinais convulsivos e em média há aproximadamente $63 \mathrm{~s}$ destes sinais por arquivo.

Como os métodos propostos possuem diversos parâmetros associados, escolheu-se arbitrariamente os seguintes valores como ponto de partida: $p=9$ para o método AR e composição de matriz de regressão; $k=m=23$ quando aplicou-se o modo inter-canais; $k=m=5$ para métodos que se utilizaram do modo intra-canal. Com estes hiperparâmetros definidos, realizou-se uma rodada inicial de testes e verificou-se que os métodos AR e de Covariância atingiram médias geométricas com valores de $92,26 \%$ e $87,69 \%$ respectivamente. Além disto, foi constatado pelos resultados obtidos, que os métodos aplicados no modo intra-canal tiveram médias geométricas superiores em comparação aos aplicados pelo inter-canais. Destaca-se também, que ao utilizar a matriz de kernel RBF, os resultados foram maiores aos obtidos pelos métodos em que a matriz polinomial foi aplicada. Porém, os resultados dos métodos propostos foram menores que os métodos utilizados como comparativos (Covariância e AR).

A partir deste levantamento preliminar, resolveu-se executar a exploração dos hiperparâmetros associados aos métodos propostos por este trabalho. Inicialmente esse processo investigativo foi realizado para a função de kernel polinomial, considerando apenas a utilização da esparsificação através do $k$-Means e pelo modo intra-canal. Portanto, buscou-se utilizar valores de $C=\{0,01, \quad 0,1,1,10,100\}$ e simultaneamente variando a ordem do polinômio com valores iguais a $\{1,2,3,4,5\}$. Nesta análise foi possível compor o gráfico da Figura 5, na qual se verifica que com o aumento da ordem do polinômio, os valores de média geométrica têm característica de queda e, portanto escolheu-se a ordem 1 para o kernel polinomial. Além disto, a partir dos resultados optouse por utilizar o valor do parâmetro $c=0,01$.

A próxima exploração de hiperparâmetros realizada, foi aplicada em $k$ e $m$ para os métodos de esparsificação e simultaneamente para o parâmetro $\sigma$ da função de kernel RBF. Nesta etapa é ressaltado que a análise foi realizada em ambos os modos de extração (inter-canais e intra-canal). Deste modo, os valores escolhidos para exploração dos parâmetros 


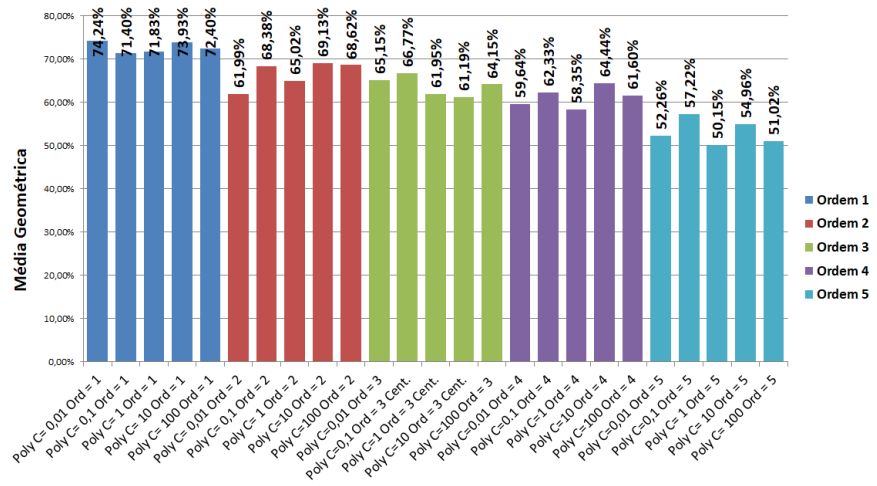

Figura 5. Exploração Kernel Polinomial
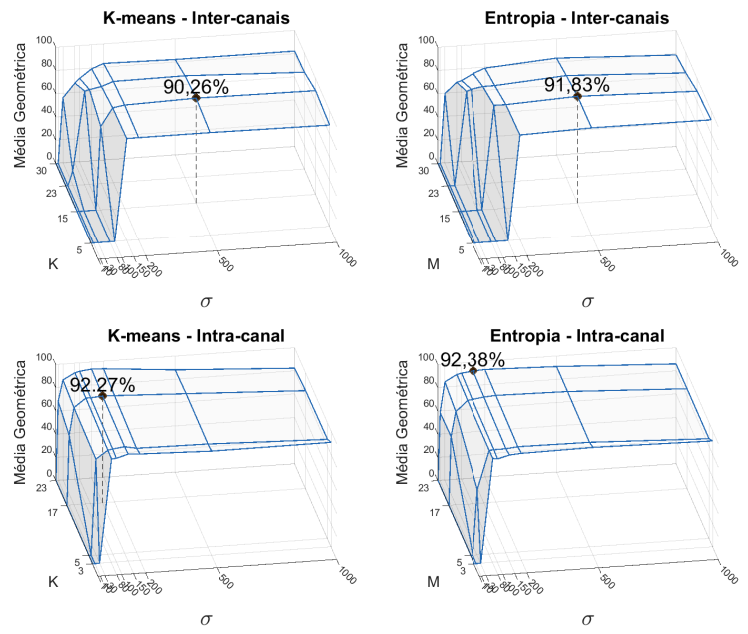

Figura 6. Mapas de superfície para exploração de hiperparâmetros.

para a análise inter-canais foram definidos de maneira que $K=M=\{5,15,23,30\}$, e para o modo intra-canal escolheuse os valores de $K=M=\{3,5,17,23\}$. O parâmetro $\sigma$ é comum aos dos grupos e optou-se por valores iguais a $\{1,10,30,80,100,150,200,500,1000\}$. Os resultados obtidos nesta etapa, são ilustrados através da Figura 6, que exibe quatro mapas de superfície destacando as maiores médias geométricas obtidas em cada análise. Portanto, com esses resultados obtidos optou-se em utilizar valores de $K=M=15$ e $\sigma=500$ para inter-canais, e para os métodos em que foi aplicada o modo intra-canais, escolheu-se valores de $K=17$, $M=23$ e $\sigma=200$.

No último processo exploratório de hiperparâmetros, fez-se também a análise dos valores de $p$. Desta maneira, identificouse que os valores de $p=8$ para o método AR e $p=5$ para composição de matrizes de regressão, fizeram com que os resultados em geral fossem aumentados. Estes são ilustrados pela Figura 7, na qual são exibidas todas as médias geométricas dos métodos aplicados no paciente chb01 e considerando a hipótese 3. Nesta Figura, destacam-se as cores de cada barra, como azuis para os métodos clássicos, roxos para os

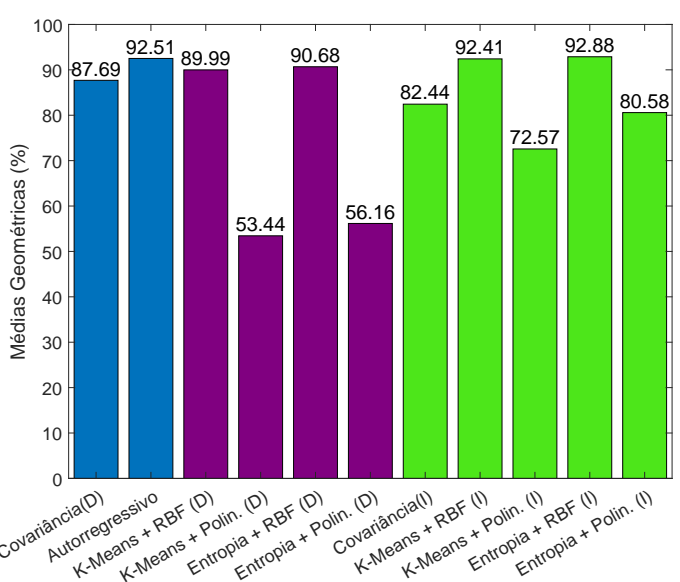

Figura 7. Resultados dos métodos para Paciente chb01 e hipótese 3.

métodos em que se utilizou o modo inter-canais e verdes para os que se assumiu o modo intra-canais. Neste último grupo, é evidenciado que os resultados obtidos são superiores aos que se utilizou da análise inter-canais. Além disto, em ambos os grupos o kernel polinomial teve um desempenho não tão promissor. Isto se difere quando se trata dos métodos pela matriz de kernel RBF, em que os resultados foram tão bons ou até superiores quanto aos métodos AR e Covariância.

Além das análises através da média geométrica, procurou-se avaliar também a capacidade dos modelos gerados ao predizer corretamente as épocas convulsivas e não convulsivas. Como os parâmetros foram encontrados, pensou-se em analisar o comportamento obtido pelas médias de acurácia, sensibilidade e especificidade para as três hipóteses do paciente chb01. Este estudo comparativo é exibido pela Figura 8, na qual é possível verificar que os resultados obtidos ao aplicar a matriz de kernel polinomial não são promissores, pois se constatam baixos valores nas métricas utilizadas. Porém, analisando os resultados obtidos no modo inter-canais e pelo método da matriz de kernel RBF, é possível verificar que as taxas médias de acurácia e especificidade possuem valores numa faixa de 90 a $95 \%$ e a sensibilidade com valores inferiores numa faixa de 70 a $86 \%$. Neste caso também pode-se realizar uma análise comparativa com o método da covariância, que obteve taxas de acurácia e especificidade similares, contudo para a sensibilidade nota-se que seus valores são inferiores.

Quando a análise deste gráfico é realizada para o grupo do modo intra-canal, pode-se salientar que nas três hipóteses, ao se aplicar os métodos que utilizam da matriz de kernel RBF os valores de sensibilidade são superiores aos demais métodos deste grupo, e estão numa faixa de 90 a $98 \%$. Além disto, ao se analisar as demais métricas para estes métodos verifica-se que as faixas de valores estão num patamar similar ao da sensibilidade. Como dito anteriormente, o método AR é utilizado para fins comparativos e pôde-se perceber que o mesmo teve um comportamento dos resultados similar ao método da covariância no grupo da análise inter-canais. 

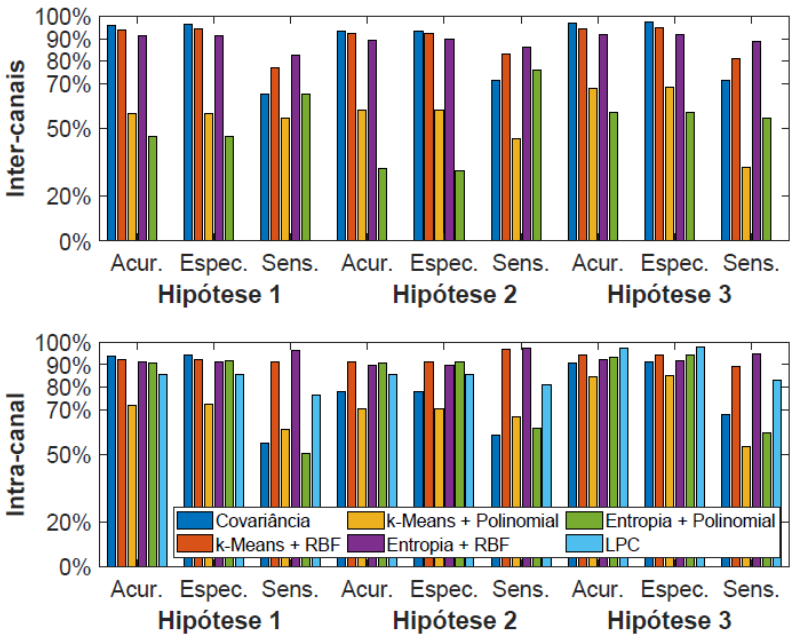

Figura 8. Métricas para chb01 todas hipóteses.

Os resultados obtidos pelos métodos da esparsificação pelo $\mathrm{K}$-means e critério de entropia associados à matriz de kernel RBF são promissores, porém, deve-se enfatizar que como a exploração dos hiperparâmetros foi realizada apenas para os sinais do paciente chb01, existe uma possibilidade de que o desempenho para demais pacientes seja inferior. Consequentemente, foi realizada a análise com outros pacientes e nesta etapa, excluíram-se aqueles métodos dos quais utilizam a matriz de kernel polinomial.

A escolha destes novos casos a serem analisados, foi realizada arbitrariamente e portanto utilizou-se dos casos chb05, chb14 e chb18. Os resultados obtidos desses pacientes são ilustrados pela Figura 9, a qual exibe as médias geométricas calculadas para os modos de extração inter-canais e intracanal, considerando também todas as hipóteses levantadas na seção III-B. Nesta figura é possível visualizar que a superioridade dos métodos de extração intra-canal se manteve. Além disto, constata-se que os resultados dos métodos propostos por este trabalho, têm um desempenho tão bom ou até melhor, com relação aos métodos utilizados como comparativos. Especificamente neste caso, destacam-se os resultados do paciente chb14, que visualmente é evidenciado a diferença de desempenho através dos gráficos.

Outra informação pertinente que pode ser descrita, é que os resultados obtidos com o paciente chb18 são no geral inferiores aos demais pacientes. Podem existir diversos motivos para essa ocorrência, porém é possível supor que os sinais tenham características de artefato ou ruído que afetam diretamente no desempenho do modelo. Outro motivo pode estar relacionado com a questão dos hiperparâmetros, que foram selecionados a partir dos resultados obtidos com o paciente chb01. Pode-se notar também nesta Figura que para este último paciente e considerando a hipótese 3 , os resultados tem uma queda de valores comparando com as demais hipóteses. A explicação disto, se dá por que utilizaram-se menos arquivos na hipótese 3 , pois o período convulsivo nesses casos terminava nos
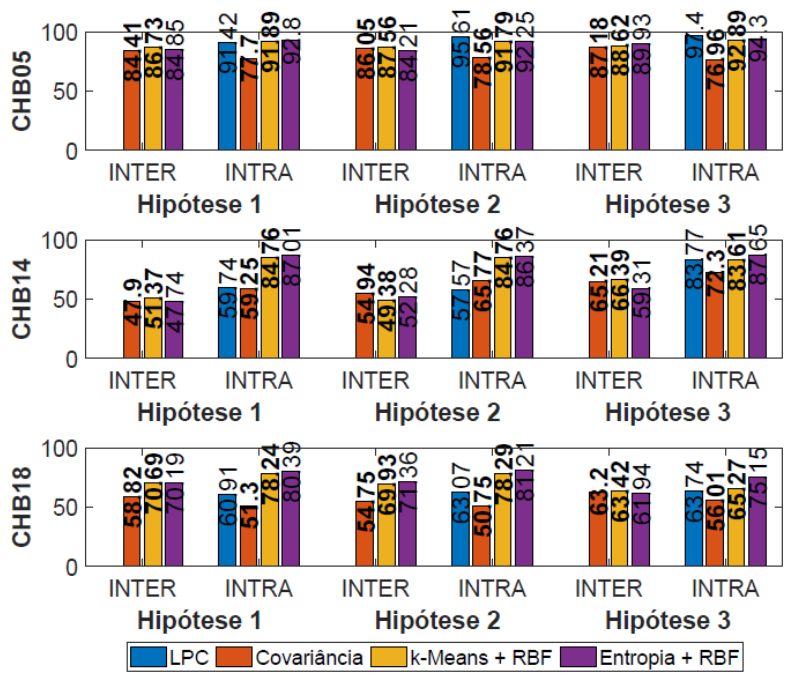

Figura 9. Resultados três pacientes.

últimos segundos da aquisição, não sendo possível aplicar os critérios estabelecidos pela Figura 2.

No intuito de realizar um estudo comparativo entre os desempenhos obtidos neste trabalho e por trabalho descritos na revisão bibliográfica, pensou-se em escolher os pacientes que um dos autores apontou como obtendo os piores resultados. Desta maneira, identificou-se que em [13], os desempenhos obtidos pelos pacientes chb02, chb05 e chb07 não foram tão satisfatórios. Nota-se que no trabalho escolhido, o autor também faz o uso do classificador OLS bem como se utiliza das mesmas métricas descritas no presente artigo, para avaliar o desempenho dos modelos. Com isto, foi possível compor a Tabela I, na qual é possível descrever que os métodos propostos por este trabalho, numa visão geral tem desempenhos maiores que os comparados. Isto é evidenciado também quando se tratam da acurácia e principalmente sensibilidade. Esta última tem um maior desempenho para todos os casos em que se utilizaram os métodos proposto pelo presente artigo. Destacando os resultados obtidos nos pacientes chb05 e chb07, pelo método de esparsificação através do critério de entropia associado à matriz de kernel, que obteve valores nas três métricas analisadas acima de $90,00 \%$. Porém, quando se trata da especificidade, o método escolhido pelo autor tem certa elevação. De acordo com os resultados obtidos no paciente chb02, supõe-se que os modelos do presente artigo, fazem uma má predição dos sinais não convulsivos, todavia isto pode estar relacionado às mesmas razões identificadas ao se analisar os resultados do paciente chb18.

\section{CONClusão.}

No presente artigo é descrito um estudo a fim de apresentar uma nova abordagem para extração de atributos de sinais EEG, com intuito de potencializar modelos que fazem o papel de identificar crises epiléticas. Inicialmente foram fundamentadas as descrições matemáticas dos métodos utilizados neste trabalho, tanto para a etapa de extração de atributos como para 
Tabela I

ESTUDO COMPARATIVO REVISÃO BIBLIOGRÁFICA

\begin{tabular}{|c|c|c|c|c|c|}
\hline СНB02 & $\begin{array}{c}\text { MFCC } \\
{[13]}\end{array}$ & Covar. & $\mathbf{A R}$ & $\begin{array}{c}\text { K-means } \\
\text { Kern. RBF }\end{array}$ & $\begin{array}{c}\text { Entropia } \\
\text { Kern. RBF }\end{array}$ \\
\hline Acur. (\%) & 96,21 & 73,47 & 61,05 & 75,09 & 68,23 \\
\hline Sens. (\%) & 37,58 & 18,05 & 77,89 & 86,83 & 96,75 \\
\hline Espec. (\%) & 99,90 & 76,27 & 59,81 & 73,67 & 66,17 \\
\hline M.G (\%) & 71,22 & 46,59 & 65,76 & 78,32 & 75,87 \\
\hline \multicolumn{6}{|l|}{ CHB05 } \\
\hline Acur. (\%) & 91,39 & 98,5 & 97,86 & 95,55 & 95,69 \\
\hline Sens. (\%) & 27,46 & 67,6 & 96,44 & 87,54 & 91,47 \\
\hline Espec. (\%) & 100,00 & 99,52 & 97,9 & 95,83 & 95,82 \\
\hline M.G (\%) & 63,08 & 87,18 & 97,40 & 92,89 & 94,30 \\
\hline \multicolumn{6}{|l|}{ CHB07 } \\
\hline Acur. (\%) & 91,97 & 96,24 & 94,61 & 95,41 & 93,85 \\
\hline Sens. (\%) & 12,94 & 73,86 & 75,77 & 86,84 & 92,34 \\
\hline Espec. (\%) & 100,00 & 96,59 & 94,91 & 95,57 & 93,86 \\
\hline M.G (\%) & 49,19 & 88,22 & 87,95 & 92,51 & 93,35 \\
\hline
\end{tabular}

classificação. Na sequência, foi explicado como o conjunto de dados escolhido é particionado a fim de tentar resolver problemas associados aos sinais transitórios pré e pós o período ictal. Além disto, levantou-se um questionamento da aplicação da extração de atributos através de dois modos configurados como inter-canais e intra-canal. Os diversos experimentos conduzidos tem o intuito de validar os métodos escolhidos e pode-se destacar inicialmente que os resultados obtidos ao se utilizar a matriz de kernel polinomial, não foram satisfatórios, em comparação ao uso matriz de kernel RBF. Esta por sua vez, quando aplicada juntamente dos métodos de esparsificação, caracterizou um alto desempenho, quando comparado aos métodos AR e Covariância. Destaca-se ainda que ao utilizar o modo intra-canal, esses resultados são superiores e com valores de $92,03 \%$ para acurácia, $91,03 \%$ para especificidade e $94,67 \%$ de sensibilidade considerando a segmentação baseada na hipótese 3 e análises realizadas nos arquivos do paciente chb01. Para um segundo experimento realizado, identificou-se que ao utilizar a esparsificação associada à matriz de kernel RBF, os desempenhos analisados para outros pacientes são promissores. Destaca-se nesta etapa resultados obtidos ao se aplicar os métodos nos arquivos do paciente chb14. Neste caso, os valores de acurácia, sensibilidade e especificidade que foram obtidos são nitidamente superiores aos resultados dos métodos utilizados como comparativo. Uma análise comparativa final foi realizada entre um dos trabalhos da revisão bibliográfica, e pôde-se verificar que os resultados de média geométrica do presente trabalho eram superiores. Destaca-se nesta etapa os resultados do paciente em que foi obtido o resultado de $94,30 \%$ ao utilizar o algoritmo da matriz de kernel RBF associada a esparsificação pelo critério de entropia. Pelos resultados obtidos, conclui-se que realizar análises intracanal aplicando o método de matriz de kernel RBF tanto com a esparsificação pelo K-means como pelo critério de entropia, tem grande desempenho na etapa de classificação. Ressalta-se ainda que apesar dos bons resultados obtidos, ainda há necessidade de outros testes como, por exemplo, uma exploração de parâmetros exclusivos aos pacientes em análise. Outro estudo que pode ser levantado é se outras funções de kernel conseguem desempenhar tão bem este papel. Portanto, é possível concluir que estes métodos descritos são promissores, e podem ser de grande auxílio no papel de extrair informações importantes de sinais de EEG.

\section{AgRADECIMENTOS}

O presente trabalho foi realizado com apoio da Coordenação de Aperfeiçoamento de Pessoal de Nível Superior - Brasil (CAPES) - Código de Financiamento 001. Os autores agradecem também à FUNCAP (processo no. BMD-000801413.01.17/20) e ao CNPq (processo no. 309379/2019-9) pelo apoio financeiro para realização desta pesquisa.

\section{REFERÊNCIAS}

[1] Rupom, F. F. et al. EMG Controlled Bionic Robotic Arm using Artificial Intelligence and Machine Learning. In: 2020 IEEE Region 10 Symposium (TENSYMP). IEEE, 2020. p. 334-339.

[2] Kang, J. et al. Transcranial recording of electrophysiological neural activity in the rodent brain in vivo using functional photoacoustic imaging of near-infrared voltage-sensitive dye. Frontiers in neuroscience, v. 13 , p. $579,2019$.

[3] Abiri, R. et al. Decoding attentional state to faces and scenes using EEG brainwaves. Complexity, v. 2019, 2019.

[4] Ramos-Aguilar, R. et al. Feature extraction from EEG spectrograms for epileptic seizure detection. Pattern Recognition Letters, v. 133, p. 202209, 2020.

[5] Fisher, R. S. et al. ILAE official report: a practical clinical definition of epilepsy. Epilepsia, v. 55, n. 4, p. 475-482, 2014.

[6] Sengur, A.; GUO, Yanhui; AKBULUT, Yaman. Time-frequency texture descriptors of EEG signals for efficient detection of epileptic seizure Brain Informatics, v. 3, n. 2, p. 101-108, 2016.

[7] Mormann, F. et al. On the predictability of epileptic seizures. Clinical neurophysiology, v. 116, n. 3, p. 569-587, 2005

[8] Baig, M. Z.; ASLAM, Nauman; SHUM, Hubert PH. Filtering techniques for channel selection in motor imagery EEG applications: a survey. Artificial intelligence review, v. 53, n. 2, p. 1207-1232, 2020.

[9] Alçin, Ö. F. et al. Multi-category EEG signal classification developing time-frequency texture features based Fisher Vector encoding method. Neurocomputing, v. 218, p. 251-258, 2016.

[10] Mo, H.; Zhao, Y. Motor imagery electroencephalograph classification based on optimized support vector machine by magnetic bacteria optimization algorithm. Neural Processing Letters, v. 44, n. 1, p. 185-197, 2016.

[11] Rahman, M. A. et al. Employing PCA and t-statistical approach for feature extraction and classification of emotion from multichannel EEG signal. Egyptian Informatics Journal, v. 21, n. 1, p. 23-35, 2020.

[12] Boonyakitanont, P. et al. A review of feature extraction and performance evaluation in epileptic seizure detection using EEG. Biomedical Signal Processing and Control, v. 57, p. 101702, 2020

[13] Silva Júnior, J. P. Métodos de extração de atributos para detecção de crises epiléticas: uma abordagem comparativa. 2019. 155 f. Dissertação (Mestrado em Engenharia de Teleinformática)-Centro de Tecnologia, Universidade Federal do Ceará, Fortaleza, 2019.

[14] Milliken, G. A.; Johnsson, D. E. Analysis of messy data, volume III analysis of covariance. Chapman and Hall/CRC, 2001.

[15] Shumway, R. H.; STOFFER, David S.; STOFFER, David S. Time series analysis and its applications. New York: springer, 2000.

[16] Al-Fahoum, A. S.; Al-Fraihat, A. A. Methods of EEG signal features extraction using linear analysis in frequency and time-frequency domains. International Scholarly Research Notices, v. 2014, 2014.

[17] Shoeb, A. H. Application of machine learning to epileptic seizure onset detection and treatment. 2009. Tese de Doutorado. Massachusetts Institute of Technology.

[18] Santos, J. D. A.; Barreto, G. A. Novel sparse LSSVR models in primal weight space for robust system identification with outliers. Journal of Process Control, v. 67, p. 129-140, 2018.

[19] Barachant, A. et al. Classification of covariance matrices using a Riemannian-based kernel for BCI applications. Neurocomputing, v. 112, p. $172-178,2013$ 